\title{
EchoGéo
}

ECHOGEO $53 \mid 2020$

Dénominations plurielles. Quand les noms de lieux se concurrencent

\section{La variation toponymique dans l'oraliture comme pratique infrapolitique : études de cas à Uchon et Paris}

Jean-Baptiste Bing

\section{(2) OpenEdition}

Journals

Édition électronique

URL : https://journals.openedition.org/echogeo/19897

DOI : 10.4000/echogeo.19897

ISSN : 1963-1197

Éditeur

Pôle de recherche pour l'organisation et la diffusion de l'information géographique (CNRS UMR 8586)

Référence électronique

Jean-Baptiste Bing, «La variation toponymique dans l'oraliture comme pratique infrapolitique : études de cas à Uchon et Paris », EchoGéo [En ligne], 53 | 2020, mis en ligne le 30 septembre 2020, consulté le 10 août 2021. URL : http://journals.openedition.org/echogeo/19897 ; DOI : https://doi.org/10.4000/ echogeo. 19897

Ce document a été généré automatiquement le 10 août 2021.

EchoGéo est mis à disposition selon les termes de la licence Creative Commons Attribution - Pas d'Utilisation Commerciale - Pas de Modification 4.0 International (CC BY-NC-ND) 


\title{
La variation toponymique dans l'oraliture comme pratique infrapolitique : études de cas à Uchon et Paris
}

\author{
Jean-Baptiste Bing
}

L'auteur remercie la DREAL Bourgogne franche-Comté qui a commandé ce travail, la municipalité d'Uchon et les Uchonais pour leur accueil et leur confiance, ainsi que Laurie Darroux qui a porté le projet Légendes d'Uchon à la MPOB, a réalisé la quasi-totalité des entretiens et a rédigé le rapport final.

\section{De l'entrée de la toponymie en légendaire}

1 La toponymie a à voir avec l'imaginaire de multiples manières; nous nous pencherons ici sur deux modalités de son rapport au légendaire : comment la toponymie entretient la mémoire d'un certain légendaire d'une part, et comment à l'inverse le légendaire peut nourrir la toponymie. Plus précisément, l'objectif de cet article est de montrer comment cette circulation entre imaginaire et lieux réels constitue une arme de résistance « infrapolitique », c'est-à-dire entretenant une résistance de basse intensité mais à long terme de la part de groupes subalternes face à des groupes puissants (ou du moins jugés tels), pouvant préparer à une résistance plus formelle (Scott, 2009, p. 199-218).

2 Nous examinerons deux cas d'étude. Le premier est celui de la microtoponymie populaire dans la commune d'Uchon (Saône-et-Loire), et plus particulièrement celle se rapportant aux chaos granitiques nombreux dans le Bois de la Ravière qui jouxte le bourg. Les cartes postales d'il y a un siècle témoignent que, à l'époque, ce plateau vallonné et ses pentes septentrionales, composés de sols acides et pauvres, étaient dominés par les landes et les pâtures ; la déprise agricole les a transformés en une forêt de feuillus (hêtraie dominante), pour laquelle les habitants expriment un fort 
attachement tant pour des raisons écologiques que par les pratiques qu'elle permet (chasse, cueillette des champignons, ressourcement...). Or ledit bois est menacé par un projet d'enrésinement, suite au rachat d'une majeure partie de sa surface par un groupe forestier connu pour ses pratiques industrielles peu soucieuses de biodiversité, et dont les habitants supposent qu'il bénéficie du soutien des autorités forestières (CRPF : Centre régional de la propriété forestière) face à une " passivité » reprochée au Parc naturel régional du Morvan et au département. Dans ce cadre, après l'échec d'autres recours (achat du site par le département...), la commune et divers collectifs habitants et associatifs ont sollicité la DREAL BFC (direction régionale de l'environnement, de l'aménagement et du logement Bourgogne-Franche-Comté) pour obtenir le classement du bois de la Ravière.

3 Le deuxième cas d'étude porte sur Paris : alors que chaque centimètre carré de la ville est cartographié, nommé, répertorié - du moins en ce qui concerne sa surface et ses parties publiques, du tréfonds du métro à l'antenne sommitale de la Tour Eiffel ${ }^{1}$ - et alors que la grande métropole fait figure d'archétype de l'hyper-modernité triomphante, technocratique et normalisatrice (Faburel 2018), le légendaire y a encore sa place. Nous nous pencherons précisément sur deux contes $^{2}$, La rue du Puits-qui-Parle (Gougaud, 1981, p. 25-31) et Rue de Bièvre (André, 2008, p. 158-161), qui font tous deux référence à des éléments de la géographie parisienne aujourd'hui rendus invisibles : la rue du Puits-qui-Parle a été rebaptisée en 1867 rue Amyot, odonyme qu'elle porte toujours, et la Bièvre, affluent de la Seine canalisé depuis plusieurs siècles, a été enterrée au XIX ${ }^{e}$ siècle et ses eaux s'écoulent depuis dans le réseau des égouts.

4 Après une présentation des récits en présence, une première partie, épistémologique et méthodologique, visera à justifier pourquoi ils sont l'objet d'une mise en regard et comment il y sera procédé; une deuxième montrera comment contes, légendes et récits oraux permettent d'entretenir un écart à la norme et à la normalisation et d'enrichir le réel quotidien; enfin la troisième s'attardera sur leur pouvoir d'encapacitation d'un groupe subalterne lors d'un rapport de force.

\section{Des récits et des lieux}

Commençons par résumer brièvement les récits en présence et, tout d'abord, les deux récits parisiens. Le premier, $L a$ rue du Puits-qui-Parle, prend place aux temps carolingiens : un jeune chevalier entretenait un grand amour avec la fille cadette du seigneur voisin qui, lui, espérait faire épouser son aînée au dit chevalier. Un jour, le vieux seigneur apprend cela - et peu de temps après la cadette disparaît. Le jeune chevalier s'en remet peu à peu et le père, à force de persévérance, obtient le mariage désiré. Mais quelques temps plus tard, le jeune couple et le vieux seigneur deviennent fous, après avoir - disent-ils - entendu une voix sépulcrale venue d'un puits de la rue les maudire. L'énigme se résout lors de la mise à sac de Paris et de ses environs par les Normands, qui découvrent la fille cadette au fond d'un cachot dont la seule fenêtre donnait sur le puits : jetée là par son père, le puits lui avait donné à entendre les conversations de son aimé et de sa sœur nonchalamment assis sur la margelle... Le second récit, La rue de Bièvre, nous est contemporain : un policier, effectuant sa ronde rue de Bièvre, y découvre un homme agenouillé à même la chaussée et en train d'ausculter icelle. Le sommant de libérer la place, il commence à converser avec l'homme qui, peu à peu, lui dévoile sa quête : retrouver, par-delà le temps et l'asphalte, 
la Bièvre disparue et les castors qui lui auraient donné son nom. Le policier se révèle un sourcier doué, qui finit par accepter son don et retourne l'exercer dans son village.

Dans le cas d'Uchon, la nature du corpus est très différente : la DREAL, pour nourrir son dossier en vue d'un examen au Ministère de l'écologie, a missionné deux cabinets d'étude pour les volets " paysagiste » et «naturaliste » et, pour le volet « légendaire ", la Maison du Patrimoine Oral de Bourgogne ${ }^{3}$. Une enquête de terrain en est sortie, dont cet article mobilisera les trois moments initiaux : deux parcours commentés, menés le 5 novembre 2019 et le 14 janvier 2020, avec à chaque fois une dizaine de personnes issues de la commune et des collectifs mobilisés, et un travail bibliographique sur des témoignages écrits issus du même milieu (Dollet-Priet et Vannier, 2003...), et constituant une partie du rapport d'étape fourni à la DREAL (Bing, 2020)4. Les deux parcours commentés avaient pour but de faire découvrir le site aux "experts" paysagistes, naturalistes et ethnographes - les habitants ${ }^{5}$ et militants engagés sur ce dossier leur servant de guide et les nourrissant donc de leur propre expertise. Si ces heures passées ensemble, sur place, ne furent pas enregistrées, elles ont permis de briser la glace - ce qui constituait un préalable nécessaire à des entretiens plus poussés. De plus les notes prises au cours de ces journées, complétées par le travail bibliographique, ont permis de dégager des grandes lignes concernant le rapport des personnes rencontrées au Bois de la Ravière - entre autres, l'entrelacement des récits et légendes avec l'expérience quotidienne du lieu. Pour résumer ces rencontres selon une grille de lecture raffestinienne (Raffestin 1995, Bing 2018: 113-115), tenants de savoirs et des territorialités «de référence » et «vernaculaires » ont pu entamer un processus de mise au point d'un langage «d'échange", avec l'objectif de se renforcer réciproquement.

\section{L'« oraliture », clé de deux « cas extrêmes »}

7 Nous aurons à faire face à deux difficultés majeures. La première tient à la nature déjà évoquée des corpus constitués, et au rapport différencié du chercheur avec eux. Dans le cas d'Uchon, il s'agit de données collectées au cours d'un processus de recherche à la méthodologie bien balisée : le corpus a été constitué en vue de l'analyse. Dans le cas de Paris, il s'agit par contre de deux textes (choisis parmi des dizaines d'autres) glanés au cours de lectures, réutilisés à l'oral dans le cadre de manifestations de " néo-contage " (Calame-Griaule, 2001) auxquelles l'auteur de ces lignes a pu participer : randonnées contées, veillées participatives, etc. Parfois ces textes ont été utilisés tels quels (sans changer les éléments non-essentiels : personnages, lieu, etc.), mais d'autres fois la raconterie et son contexte ont suscité une adaptation. Au final, d'une part les deux récits préexistent à l'analyse, d'autre part ils ne sont pas forcément représentatifs de l'ensemble des contes et légendes existant mettant en scène la toponymie à Paris ou ailleurs. Cependant, nous faisons le pari que le rapprochement des deux corpus relève d'un «bricolage» (Lévi-Strauss, 1962), qui se révélera utile pour trois raisons: épistémologique, méthodologique, heuristique. Tout d'abord, il fait sens pour le praticien de $l^{1}$ « oraliture ${ }^{6}$ » qu'est le géographe et conteur auteur de ces lignes, qui s'identifie à un «territoire de parole » (Darroux, 2011) reliant (entre autres) le Morvan où il vit et la capitale d'où il vient, travaillant et contant entre les deux. Cette posture épistémologique certes bâtarde autorise quelque regard scientifique, sous réserve de deux impératifs méthodologiques: l'intersubjectivité d'abord, d'où l'importance de 
croiser les regards tant concernant l'échange des récits que la construction de la démarche de recherche ; une mise en miroir des deux corpus, visant non point à une comparaison, mais à un simple éclairage réciproque des deux situations (méthode détaillée dans Bing, 2018, p. 67-105). Enfin ce bricolage a une utilité heuristique en permettant de discuter d'une nouvelle façon des hypothèses déjà travaillées dans un cadre plus académique.

8 La première des hypothèses que nous explorerons ici porte sur l'interaction entre les récits légendaires, source essentielle du lien au «territoire du quotidien " (Raffestin, 1995), et l'infrapolitique. En un sens, ces récits peuvent fournir une ressource lors de confrontations avec les autorités "de référence» (id.), que ce soit sur le mode de l'affrontement (dans le cas d'Uchon, avec le propriétaire légal du bois de la Ravière et ses soutiens institutionnels) ou sur le mode de la collaboration (avec la DREAL). Réciproquement, une situation de conflit (y compris sur le mode latent de l'infrapolitique) peut contribuer à revitaliser des récits autrefois oraux figés par l'écrit, et les faire revenir dans le champ du dire direct. D'où une conséquence à propos du concept d'«oraliture »: alors qu'il a émergé à propos de sociétés dites "à tradition orale » (afin de conférer aux pratiques de l'oralité une dignité qui n'était ordinairement accordée qu'aux pratiques issues de l'écriture, et de pallier les insuffisances de la notion de "littérature orale »), nous entendons montrer son utilité dans les pays où, en dépit d'une supériorité conférée et reconnue à l'écrit, l'oralité n'a jamais disparue (à condition, toutefois, de ne pas considérer l'oraliture comme forcément opposée à l'écrit, mais comme son complément) - en l'occurrence pour appréhender des résistances infrapolitiques en France (Darroux, 2011).

9 La deuxième difficulté tient à la dissemblance entre Uchon et Paris, qui constituent en effet deux «cas extrêmes » (Flyvbjerg, 2011) dans le contexte de l'aménagement du territoire français, tant ces communes se situent aux deux extrémités en terme de poids de la population: d'un côté, la capitale millionnaire, épicentre de l'un des États historiquement les plus centralisés du globe; de l'autre, une commune hyperrurale, comptant une centaine d'habitants et située à la marge tant des territoires dont elle relève (département de Saône-et-Loire, PNR du Morvan, Communauté de communes du Grand Autunois-Morvan) que des grands axes de communication les plus proches (lignes SNCF Paris-Lyon et Nevers-Dijon, axe routier de la vallée de l'Arroux...). Nous faisons ainsi le pari que la disproportion même de ces deux cas se révélera heuristique, en considérant que la division canonique entre urbain et rural demeure certes structurante, mais selon des modalités qui demandent à être repensées selon de nouveaux paradigmes (Cornu et Delfosse, 2017 ; Debarbieux, 2007). Que les différentes déclinaisons de l'oraliture (située aux marges des productions culturelles tant elle relève à la fois des «arts de la parole » et de la mise par écrit, de la spontanéité du discours et du travail d'artistes professionnels) permettent d'appréhender ou, du moins, d'explorer cette relativité de la division entre rural et urbain (notamment dans leurs marges, sociales et/ou spatiales où se manifeste l'infrapolitique) est donc la deuxième hypothèse que nous entendons tester. 


\section{Cultiver le mystère face à une normalisation qui aplatit le réel}

10 Le conte La rue du Puits-qui-Parle contribue de plusieurs manières à réintroduire du mystère, de l'incertitude, dans des rues de Paris pourtant fort aménagées et bien connues, en autorisant un balancement subtil, propice à un jeu de rationalités variées (Stoczkowski, 1999), entre savoir et croyance et entre réel et fiction (Bing 2016). Tout d'abord, à l'instar de la quasi-totalité des contes et légendes (Gougaud, 2008), cette histoire compte plusieurs versions : sur un même canevas de base les habillages sont nombreux ${ }^{7}$. De plus, si la rue Amyot (ancienne rue du Puits-qui-parle) est bien identifiée depuis le $\mathrm{XVI}^{\mathrm{e}} \mathrm{s}$. (son tracé actuel figure déjà sur des plans de cette époque), la légende relate un fait bien plus ancien, puisque remontant aux temps carolingiens. Deuxième facteur d'incertitude, donc : ces temps demeurent assez mal connus du grand public en dehors de quelques images d'Épinal sur les rois francs et les ravages des Normands.

11 Si l'on s'en tient à ces seuls faits, la dimension infrapolitique demeure assez négligeable - l'urbanisme ayant en effet assez massivement assimilé nombre d'odonymes faisant référence à un passé ancien plus ou moins imaginaire : rue des Quatre fils Aymon, rue Boutebrie, etc. Cependant la survie de certains toponymes disparus participe, avec d'autres manifestations puisant elles aussi dans les images tirées d'un passé ancien plus ou moins conforme au réel, à faire vivre des petits groupes contre-culturels dont l'action relève directement de l'infrapolitique, et qui cultivent des pratiques d'oraliture variées. Premier exemple: les Block Parisii (Paris-Saint-Germain) et Ultras Lutetia (Paris FC), qui revendiquent comme nombre de groupes de la même mouvance un rapport politique et territorial au football, exprimé par des banderoles, des tags... (Bing, 2019) ou des chants inspirés du répertoire populaire ${ }^{8}$. Deuxième exemple, dans le domaine de la chanson, justement : de nombreux textes contemporains mobilisent des figures et des moments légendaires de Paris, en les réactualisant au vu des préoccupations du moment; citons par exemple la Commune et les communards, revendiqués tant par la mouvance anarchiste (Renaud...) que par la mouvance identitaire (Vae victis...). Pour en revenir à La rue du Puits-qui-parle, si ce récit prend place parmi les «légendes noires » au sujet du Moyen-Âge et des Vikings et entretient ainsi à leur sujet l'imaginaire négatif qui sert l'idéologie de la modernité (alors que ces fantasmes sont assez largement déconstruits par les historiens contemporains), il contribue aussi à ouvrir un champ d'expression à l'imaginaire. Or celui-ci porte une potentialité subversive non-négligeable, qu'Henri Gougaud assimile à l'un des aspects de la «philosophie artisanale» que porte le conte, par exemple quand des versions nouvelles ou peu diffusées d'un conte ou d'une légende renversent le message sousjacent porté par une version canonique (Gougaud 2008).

12 Autre subversion possible : remettre à l'honneur des noms a priori anodins qui, si l'on fouille dans les arcanes de l'étymologie, se révèlent porteurs de phénomènes sociaux peu conformes aux valeurs que notre société cherche à montrer d'elle-même. Se réfèrent ainsi à l'intense activité de prostitution qui y régna trois noms de rues qui, aujourd'hui, apparaissent plutôt neutres : la rue du Petit-Musc et la rue du Pélican se dénommaient autrefois «Pute-y-muse » et «Poil-au-con»; quant à l'ex-rue Troussenonnain, elle a été doublement recouverte: d'abord par déformation en «rue Transnonain ", puis par absorption de celle-ci par l'actuelle rue Beaubourg (Hagège, 
2009, p. 231). À en croire le même auteur cet « oubli des gaillardes réalités » du passé relève, au moins en partie, d'une entreprise délibérée de « censeurs pudibonds » (id.); cependant la mémoire et les pratiques populaires continuent de porter cette mémoire du lieu. On peut lire ainsi sur Wikipédia, qui constitue un point de contact fécond d'interfécondation entre "savoirs de référence » et "savoirs vernaculaires » (Bing, 2018), au sujet de la rue Pélican :

« en 1792, elle fut appelée "rue Purgée" lorsque les prostituées furent chassées de la rue. Jean de La Tynna indique qu'elle ne méritait guère ce nom, des filles publiques continuant à l'habiter!» et que "Dans les premières pages de Guignol's Band de Louis-Ferdinand Céline, un souteneur, désireux d'indiquer vers 1914 ou 1915 qu'il ne peut gérer qu'un nombre limité de prostituées, insiste sur le fait qu'il "n'est pas le Pélican" »".

13 L'encyclopédie en ligne transmet ainsi cet imaginaire historique, à travers les références à une période de grands bouleversements toponymiques et à un auteur connu pour son usage d'un langage puisant largement dans le registre de l'argot (Calvet, 1993).

Enfin l'application du programme donné par Augustin Berque à la mésologie («renaturer la culture, reculturer la nature»: Berque, 2014) porte lui aussi une dimension politique. À Uchon, le conflit autour du bois de la Ravière semble opposer deux conceptions des relations de la "culture» à la "nature»: d'un côté, une dimension purement utilitariste, qui préside à l'extension des monocultures (résineux dans le Morvan); de l'autre, une conception plus mésologique, qui tente de tenir compte de la complexité de la relation trajective. La dimension historique, anthropique, de la «nature » n'y est pas niée. Loin de tomber dans le lieu commun qui ferait de la forêt de feuillus de la Ravière un lieu ancestral remontant aux Gaulois, la quasi-totalité des habitants rencontrés mettent en avant l'historicité du milieu: en alignant les cartes postales vieilles de plus d'un siècle, ils montrent que ce qui est aujourd'hui une forêt était alors une lande et des pâtures; ils n'en soulignent pas moins la richesse écosystémique du lieu et la force des pratiques sociales qui s'y tiennent. Enfin, dans le cas du conte de la rue de Bièvre, la recherche de la rivière et de sa faune perdues entre en résonance avec des initiatives associatives, qui visent à la redécouvrir, voire à procéder - à rebours de ce qui a été fait depuis des siècles - à un processus de «renaturalisation $»^{10}$.

15 À travers l'interrogation sur le soubassement naturel même de la ville, du village et de la commune qui les incarnent ; à travers les plongées dans un passé toujours présent ; à travers enfin les rapports subtils entre faits, savoirs et imaginaires qui sont les moteurs de la saisie de la microtoponymie et de l'odonymie par l'oraliture, se produit au final non seulement un entretien du mystère face une norme aplatissant le réel, mais aussi un enrichissement dudit réel.

\section{Le pouvoir de nommer (ou pas) : la microtoponymie comme rapport de force}

Le rapport de force qu'est la politique appelle des réponses spécifiques à chaque cas ; le jeu autour de l'incertitude et de la pluralité comme arme de lutte se décline donc d'une manière différente dans les cas parisien et uchonais. En ce qui concerne les rochers et amas granitiques du Bois de la Ravière, cela passe par l'absence de toponymes reconnus 
et partagés : en effet si les plus gros chaos et ceux ouvrant sur les points de vue les plus pittoresques, assez largement recensés avec l'expansion du tourisme dès le début du $\mathrm{XX}^{\mathrm{e}} \mathrm{s}$., bénéficient d'une toponymie bien fixée (la Griffe du diable, la Grotte de Mandrin, le Rhinocéros ${ }^{11}$...), ce n'est pas le cas de ceux situés dans la majeure partie du Bois de la Ravière - moins spectaculaire, moins touristique, et donc relevant d'une toponymie plus intimement uchonaise. Lors de la visite du 5 novembre 2019 effectuée avec la représentante de la DREAL, des membres du conseil municipal et des habitants et militants impliqués, il est apparu par exemple que le même nom («Roche fendue ») désignait, selon les personnes, deux chaos situés à quelques centaines de mètres l'un de l'autre. De nombreux autres amas et rochers isolés, moins remarquables, n'ont pas (pas encore ?) de nom reconnu par la collectivité habitante et/ou par les autorités, et sont nommés au coup par coup par chacun, selon ses références propres et selon ce qui interpelle dans le rocher. La forme d'un rocher, par exemple, qui a servi à nommer le bien identifié et dûment classé « Rhinocéros » dans les rochers de Carnaval, a inspiré au maire un "requin" évoqué en novembre; deux mois plus tard, lors d'un autre entretien collectif itinérant (sans représentant de la DREAL cette fois-ci), c'est à plusieurs qu'a été informellement désigné un " cachalot ». Il était parfois difficile, lors de ces parcours où les discussions étaient fort décousues, de faire la part du premier et du second degré, du sérieux, de l'ironie voire de la provocation: l'on passait, en quelques minutes, d'une réflexion sur la richesse écologique du site à la proposition d'établir une ZAD à proximité d'un vieux moulin hydraulique, puis à un historique du lieu - labyrinthe discursif qui obligeait à prendre un certain recul, ainsi que des pincettes, pour saisir les propos, d'autant que l'ironie constitue une arme rhétorique quand des citoyens se trouvent face à des représentants d'une autorité quelconque (DREAL et ses missionnaires, par exemple).

17 La logique de l'argot, du langage réservé aux seuls initiés, n'est pas absente de ce qui se passe à Uchon : si le savoir concernant les lieux classés et fléchés (rochers de Carnaval, chemins balisés, patrimoine bâti...) est à disposition de tout visiteur intéressé, celui concernant les lieux fréquentés par les habitants n'est - à l'instar des meilleurs coins pour cueillir les champignons - pas dévoilé au premier venu... Ces noms incertains ont aussi une part de secret, qu'il ne convient de lever que si besoin est - ce qui fait l'objet d'autres débats : la protection du site passe-t-elle forcément par la protection, si oui le classement implique-t-il forcément le tourisme, si oui quel tourisme développer, etc. Choisir de jouer ainsi sur les registres de discours, jongler (ou non) avec les toponymes, décider de les faire connaitre (ou non) et de présenter (ou non) les modalités qui ont présidé à ces choix - tout cela revient à affirmer un pouvoir (ici, celui de la collectivité habitante) à la fois sur un lieu (le Bois de la Ravière et, plus largement, le territoire communal) et à revendiquer une participation sur les politiques qui s'y expriment. Face aux « experts » qui, d'un côté comme de l'autre (MPOB et cabinets d'étude mandatés par la DREAL pour nourrir sa demande de classement d'une part, exploitant forestier et ses appuis institutionnels et économiques de l'autre) affirment leur compétence, est avancée une autre légitimité : l'expertise classique ayant tendance à fragmenter le réel de manière réductionniste, les habitants mobilisent une expérience plus intuitive et plus holistique. Celle-ci n'est d'ailleurs pas contradictoire avec toute expertise et peut éventuellement la compléter et la renforcer (Claessens, 2013). Dans le cas parisien, les groupes contre-culturels évoqués (ultras des stades, chanteurs se voulant contestataires de gauche comme de droite ou par-delà cette bipartition...) procèdent 
d'une manière proche, et tirent leur légitimité sur la ville de leur contact quotidien avec les lieux (Bing, 2019).

\section{Ouverture en forme de nuage}

Il va de soi que cet article n'a permis, au mieux, que de défricher des pistes de réflexion plus qu'il n'a démontré solidement la validité des hypothèses posées ci-dessus. Cependant, deux points saillants émergent.

19 Certes nous avons bien plus à faire dans le cas parisien à des contes et légendes déjà figés et mis par écrit qu'à des récits transmis oralement et/ou élaborés par une pratique quotidienne du lieu. Cependant, d'une part certains de ces récits étaient à l'origine (avant d'être transformés par la transmission orale puis figés par l'écrit) très proches de nos très contemporaines et bien vivantes « rumeurs urbaines " (Renard, 2006); d'autre part ils prennent places aux côtés d'autres pratiques qui, elles, sont encore bien vivantes, informelles et imprégnées d'oralité (raconteries, musiques populaires, tags...) et auxquelles Henri Gouraud relie explicitement les contes présentés dans son recueil (Gouraud, 1981, p. 21-22). Les récits entendus au bois de la Ravière d'Uchon entrent dans cette catégorie : ils n'apparaissent pas d'emblée comme un tout cohérent, structuré, mais ils forment des résurgences qui témoignent d'un rhizome sous-jacent, légendaire et concret à la fois. Se dessine donc un faisceau de pratiques culturelles, dans lequel les deux contes mis par écrit à Paris et les bouts de récits légendaires entendus à Uchon exprimeraient donc une facette possible de résistance face à une pratique technocratique et, finalement, déshumanisée et aliénante de la gestion de l'espace par une autorité publique qui tend à normer et à formaliser tous les aspects de la vie collective.

Dans le cas de la nomination des lieux et des conflits qu'elle suscite ou pour lesquels elle constitue une arme parmi d'autres, il est bien connu que la toponymie officielle offre $\mathrm{au}(\mathrm{x})$ pouvoir(s) une occasion de se donner à voir et d'afficher un récit collectif et une histoire du lieu propices à l'auto-légitimation et conformes à ses (leurs) valeurs. Mais face à ces territorialités « de référence ", et en retour, se déploie une nébuleuse de noms et de territorialités vernaculaires où la pluralité fait loi. Que ce terme de nébuleuse rejoigne étymologiquement le modèle proposé par Debarbieux (2007) pour repenser les rapports ville/campagne n'est sans doute qu'anecdotique; l'est sans doute moins le fait que les « champs médiaux » (Bing, 2018, p. 62-65) se déploient dans des marges sociales (à Paris) et spatiales (à Uchon) qui, en milieu urbain comme en milieu rural, relèvent de dynamiques sinon communes, du moins pouvant être mises en regard, questionnées et finalement mobilisées de conserve par les habitants d'un «territoire de paroles» en recherche d'encapacitation.

21 La souplesse des formes d'expression prise par l'oraliture, sa pluralité intrinsèque - qui fait coexister légendes urbaines, récits du quotidien, vieux contes mis par écrit retrouvant une vie orale enrichie... - apparaissent ainsi fondamentales, puisque c'est elles qui, en maintenant de l'informel, ouvrent des champs propices à l'expérimentation sociale et à l'encapacitation populaire pour créer de nouvelles marges à l'écart de la norme. 


\section{BIBLIOGRAPHIE}

André P., 2008. Contes des sages au fil de l'eau. Paris, Seuil, 189 p.

Aubrit J.P., 2002. Le conte et la nouvelle. Paris, A. Colin, 191 p.

Berque A., 2014. Poétique de la Terre. Histoire naturelle et histoire humaine, essai de mésologie. Paris, Belin, $238 \mathrm{p}$.

Bing J.B., 2016. Aperçu sur la genèse d'une chôra : Libertalia et la Lémurie, de la littérature au mythe. Annales de géographie, vol. 709-710, p. 272-289.

Bing J.B., 2018. Dynamiques des savoirs et co-construction des sylvosystèmes agroforestiers en pays javanais et betsimisaraka. Thèse de doctorat en sciences de la société mention géographie, Université de Genève, 366 p. URL : file:///c:/Users/UTILIS 1/AppData/Local/Temp/ unige_103460_attachment01.pdf

Bing J.B., 2019. Des tribunes aux murs des kampung indonésiens : quand les ultras marquent le territoire. Néotoponymie/Neotoponymy [En ligne]. URL : https://neotopo.hypotheses.org/2199

Bing J.B., 2020. Légendes d'Uchon : petit tour bibliographique..., Rapport d'étape à la DREAL, disponible sur demande à la MPOB.

Calame-Griaule G. (dir.), 2001. Le renouveau du conte. Paris, CNRS éd., 449 p.

Calvet L.J., 1993. L'argot en vingt leçons. Paris, 214 p.

Cornu P., Delfosse C., 2017. Marges géographiques, marges scientifiques ? Contribution ruraliste à une approche réflexive des enjeux théoriques et sociopolitiques de la territorialité. Bulletin de l'association de géographes français, vol. 94, nº 3, p. 454-471.

Darroux C., 2011. La vieille femme salie : récit d'une résistance à la modernité: Morvan $\mathrm{XX}^{e}-\mathrm{XXI} I^{e}$ siècles. Thèse de doctorat en anthropologie, Université d'Aix-Marseille 1.

Darroux C., 2017. La Maison du patrimoine oral de Bourgogne : actualité de l'activité patrimoniale et action citoyenne en région. In Situ, vol. 33, 25 p.

Debarbieux B., 2007. Le syndrome de Moctezuma, ou Réflexions sur l'actualité et la pertinence du couple ville-campagne dans l'analyse territoriale. In Monteventi Weber L., Deschenaux C., Michèle Tranda-Pittion M. (dir.), Campagne-ville : le pas de deux. Enjeux et opportunités des recompositions territoriales, Lausanne, Presses Polytechniques Universitaires et Romandes, 20 p. URL : file:///C:/Users/UTILIS 1/AppData/Local/Temp/unige_29338_attachment01.pdf Dollet-Priet L., s.d. Uchon terre d'émotions. Auto-édition.

Faburel G., 2018. Les métropoles barbares. Démondialiser la ville, désurbaniser la Terre. Lyon, Le passager clandestin, $368 \mathrm{p}$.

Flyvbjerg B., 2011. Case study. In Denzin N.K., Lincoln Y.S. (éd.), The Sage Handbook of Qualitative Research, Thousand Oaks, Sage, p. 301-316.

Gougaud H., 1981. La rue du puits qui parle. Contes de Paris. Paris, Gallimard, 159 p.

Gougaud H., 2008. Le rire de la grenouille. Petit traité de philosophie artisanale. Paris, Carnets Nord, $181 \mathrm{p}$.

Hagège C., 2009. Étymojolie. In Dictionnaire amoureux des langues, p. 227-234.

Lapouge G., 2009. La légende de la géographie. Paris, A. Michel, 275 p. 
Lévi-Strauss C., 1962. La pensée sauvage. Paris, Plon, 389 p.

Raffestin C., 1995. Langue et territoire. Autour de la géographie culturelle. In Walty S., Werlen W. (éd.), Kulturen und Raum: theoretische Ansätze und empirische Kulturforschung in Indonesien. Festschrift für Professor Albert Leemann, Zurich, Rüegger, p. 87-104.

Renard J.B., 2006. Rumeurs et légendes urbaines. Paris, PUF, 127 p.

Scott J.C., 2009. La domination et les arts de la résistance. Fragments d'un discours subalterne. Paris, éd. Amsterdam, $269 \mathrm{p}$.

Stoczkowski W., 1999. Des hommes, des dieux et des extraterrestres. Paris, Flammarion, 474 p.

Urbain J.-D., 2003. Ethnologue mais pas trop... Ethnologie et proximité, voyages secrets et autres expéditions minuscules. Paris, Payot, $285 \mathrm{p}$.

Vannier J., 2003. Uchon pêle-mêle. Auto-éd., 200 p.

\section{NOTES}

1. Les carrières qui s'étendent dans les profondeurs du sous-sol parisien demeurent, pour partie, mal connues - et sont le théâtre d'opération d'une autre pratique contre-culturelle (qui pourrait être analysée sous l'angle infra-politique) : la cataphilie (Urbain 2003).

2. Les théories littéraires distinguent assez finement le conte de la légende et d'autres genres apparentés (nouvelle fantastique, fable, etc.), en fonction de différents critères. Ainsi le conte serait situé hors du temps et de l'espace et ses personnages seraient des archétypes, tandis que d'autres genres (dont la légende) mettraient en scène des personnes, des faits, des lieux ayant pu avoir une existence historique, quoiqu'en les déformant (Aubrit, 2002). Cependant, dans la pratique, l'assimilation des deux genres est courante : s'il existe des textes correspondant assez bien aux idéaux-types, bon nombre d'histoires se situent en fait dans un entre-deux ( $L a$ rue du Puits-qui-parle en est un exemple, mais d'autres récits de Gougaud, 1981 conviendraient autant).

3. Association loi 1901, la МРОВ a pour mission de recueillir, étudier et valoriser les manifestations des cultures populaires (contes, récits de vie, langues régionales, chants, musiques, danses...) de Bourgogne (Darroux, 2017). Elle a obtenu en 2019 pour son projet de «Fabrique sociale orale» fondé sur la recherche-expérimentation et l'action sociale, le label Ethnopôle par lequel le Ministère de la Culture confère à des institutions de statuts divers, et situées dans toute la France, un rôle de centre de ressource, de médiation et d'acteur direct pour la recherche en ethnologie et en en sciences humaines.

4. Des entretiens individuels approfondis et des veillées collectives devaient suivre, et permettre grâce à des enregistrements de constituer des archives orales qui devaient compléter le fond de la MPOB (http://patrimoine-oral.org/dyn/portal/index.seam?page=home\&fonds=1). Initialement prévus au printemps et à l'été 2020 , ils ont dû être repoussés suite à la crise sanitaire et ne peuvent donc être mobilisés pour cet article. Ils ont été réalisés par Laurie Darroux, chargée de mission en ethnologie à la MPOB.

5. Certains de ces habitants vivent à l'année à Uchon, d'autre y disposent de résidences secondaires. Tous cependant revendiquent plusieurs décennies de présence dans la commune, et considèrent cette différence de mode d'habiter comme secondaire par rapport à l'attachement partagé au lieu.

6. Ce «mot-valise forgé à la fin $d u X X^{\mathrm{e}} \mathrm{s}$., désigne à la fois un produit et une production communautaire et populaire caractéristique de sociétés à tradition dite orale " (cf. https:// www.fabula.org/actualites/l-oraliture-haitienne-identite-s-structure-s-memoire-s-etrepresentations-travers-le-prisme-des_86510.php). 
7. Cf. http://www.parislenezenlair.fr/actualites/item/1090-la-legende-du-puits-qui-parle-de-larue-amyot.html.

8. Sur ces deux groupes, voir https://stadito.fr/2018/12/04/le-block-parisii/ et https:// www.vice.com/fr/article/jm7784/dans-les-tribunes-du-paris-football-club-hugo-aymar. propos de la dimension infra-politique du mouvement ultra, voir Bing, 2019.

9. Cf. https://fr.wikipedia.org/wiki/Rue_du_P\%C3\%A9lican (consulté le 11/02/2020).

10. Cf. par exemple http://www.bievre.org/ et http://marche.bievre.org/ (consultés le 25/02/2020).

11. Ces amas dominent la vallée de l'Arroux, et se situent à quelques kilomètres du Bois de la Ravière, au sud de la commune. C'est principalement là que s'est développé le tourisme à Uchon, et qu'il se concentre toujours principalement.

\section{RÉSUMÉS}

Cet article s'attache à montrer comment l'oraliture, en pérennisant l'actualité de variation toponymique, constitue une arme de résistance et d'encapacitation relevant de l'« infrapolitique ». Pour ce faire, elle s'attache à deux cas d'étude : la microtoponymie appliquée au quotidien par les habitants du village d'Uchon (Saône-et-Loire) et transmise via des récits lors de parcours commentés ; l'odonymie de deux rues de Paris, transmise à travers deux récits. Après avoir présenté les cas d'étude et le corpus, la première partie pose quelques considérations épistémologiques et méthodologiques sur ceux-ci, la manière de les traiter et les limites des résultats qui peuvent en être tirés. Puis la deuxième partie montre comment contes, légendes et récits oraux permettent d'entretenir un écart à la norme et à la normalisation et d'enrichir le réel quotidien. Enfin la troisième s'attarde sur le pouvoir d'encapacitation que représente cette oraliture pour un groupe subalterne lors d'un rapport de force. L'article conclue sur l'importance de la variante dans l'oraliture comme interstice où peuvent s'imaginer et s'incarner des résistances infrapolitiques par-delà les différences de contexte entre une métropole et un village de l'hyperruralité.

\section{INDEX}

Mots-clés : Oraliture, infrapolitique, toponymie, Uchon, Paris

Thèmes : Sur le Champ - Sur le Terrain

\section{AUTEUR}

\section{JEAN-BAPTISTE BING}

Jean-Baptiste Bing, jeanbaptiste.bing@mpo-bourgogne.org, Maison du Patrimoine Oral de Bourgogne. 